\title{
Reflecting on integrated assessment in the Socially Inclusive Agricultural Intensification (SIAGI) project
}

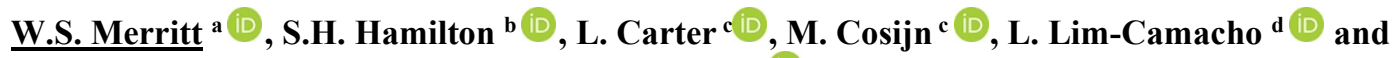 \\ C.H. Roth ${ }^{c}$ iD \\ ${ }^{a}$ Fenner School of Environment and Society, The Australian National University, Australian Capital \\ Territory, ${ }^{b}$ School of Science, Edith Cowan University, Joondalup, Western Australia, ${ }^{c}$ CSIRO Land \& \\ Water, Brisbane, Queensland, ${ }^{d}$ CSIRO Agriculture and Food, Queensland \\ Email:wendy.merritt@anu.edu.au
}

\begin{abstract}
Agricultural aide interventions are often intended to help small and marginal farmer households increase their agricultural production, and by doing so, better meet their household needs and improve their social and economic standing. However, intensifying their agricultural production requires them to have the capital assets (financial, human, natural, physical and social) and agency to access and use more agricultural inputs, develop and manage the necessary farm or community level infrastructure (e.g. for irrigation, or postharvesting), and make informed crop and land management choices. Many small and marginalised farmers are lacking in both capital and agency, which constrains their capacity to engage in, and benefit from, agricultural intensification. In this paper, we reflect on our integration research in the 'Promoting Socially Inclusive and sustainable Agricultural Intensification in West Bengal and Bangladesh' (SIAGI) project, focusing on the learnings and outcomes of being socially inclusive in our modelling practices. The Ethical Community Engagement (ECE) ethos and practice to which the SIAGI project team has committed has shaped the content of the integrated assessment frameworks that we have developed as well as the process (Figure 1) we used to develop them. Social inclusion is both a core value of the SIAGI project and an outcome against which the impact of the project will be measured.
\end{abstract}

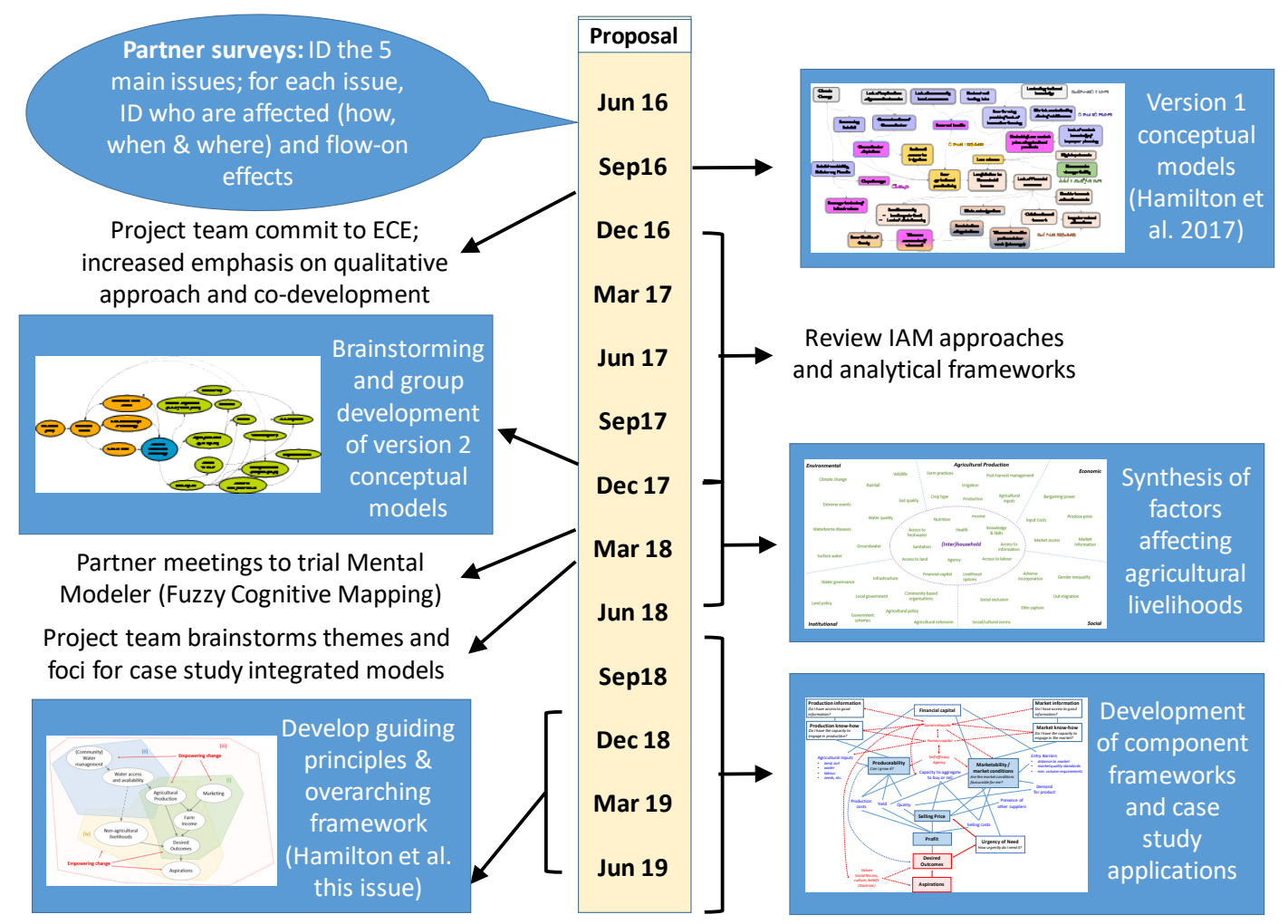

Figure 1. Timeline of key IA activities and outputs in the SIAGI project over the first three (of four) years. Timeline graphics are included for pictorial purposes only.

Keywords: Social inclusion, transdisciplinary research, research for development (R4D), Integrated assessment (IA) frameworks, ethical community engagement (ECE) 


\section{INTRODUCTION}

Sustainable agricultural intensification in developing countries is often proposed as a central means of improving the social and economic conditions of poor small and marginal farmer households. Opportunities to intensify agriculture - for example, through productivity or resource-use efficiency gains, diversification or greater cropping intensity - may necessitate the use of more agricultural inputs, development of irrigation infrastructure, and changes in how people farm or market their produce. The constraints that small and marginalised farmers face should they wish to make such changes can mean they are often unable to benefit from such improvements, either due to exclusion or from adverse inclusion.

A project on Socially Inclusive Agricultural Intensification (SIAGI, https://siagi.org/about-siagi/) has been codeveloping with communities and stakeholders, opportunities for intensifying agriculture that actively include and benefit marginalized groups. We reflect in this paper on our integration research in a project that holds social inclusion as both a core value and an outcome against which project impact will be evaluated. Section 2 provides an overview of SIAGI, defines what the project team considers by 'inclusion', and introduces the Ethical Community Engagement (ECE) ethos and practice to which the team has committed. We then outline the planned role and process of the integration research and how this activity evolved over time in response to both the ECE commitment and the developing insights from the broader SIAGI team (Section 3). In Section 4, we reflect upon the learnings and outcomes of being socially inclusive in our modelling practices.

\section{THE SIAGI PROJECT}

\subsection{Background}

SIAGI is part of a group of three projects ('sister projects') that span the Eastern Gangetic Plains of West Bengal and southern coastal zone of Bangladesh, which are funded by the Australian Centre for International Agricultural Research (ACIAR). The projects are working together to enhance the livelihoods of poor and marginalised farmers through a range of agricultural intensification strategies. SIAGI works in six study villages: two villages around Cooch Behar in West Bengal, two villages in the coastal zones around Khulna and Patuakhali in southern Bangladesh, and two villages in the Bankura District of West Bengal. Table 1 outlines the objectives and activities from the project proposal.

Table 1. Objectives and key activities from the SIAGI project proposal.

\begin{tabular}{|l|ll|}
\hline Objective & Key & Activities \\
\hline 1. To understand how key & 1.1 & Household typology and livelihood \\
social, institutional, economic & 1.2 & Value chain analysis \\
and environmental factors affect & 1.3 & Social inclusivity and equity issues \\
livelihood risks, social & 1.4 & Visions, aspirations, livelihood potentials and choices of farmers \\
exclusion, adverse & 1.5 & Policy analysis and institutional mapping \\
incorporation and & 1.6 & Environmental and climatic characterisation \\
environmental degradation in & 1.7 & Practices in achieving social inclusion, resource mobilisation and technology \\
agricultural intensification & 1.8 & Integrated risk assessment based on above activities \\
\hline 2. To identify opportunities to & 2.1 & Conceptualise socio-ecological system \\
manage risk and promote social & 2.2 & Develop and test integrated assessment model(s) \\
inclusivity and equity under & 2.3 & Conduct scenario modelling and trade-off analysis \\
different agricultural & 2.4 & Synthesise key insights \\
development scenarios using & & \\
scenario and trade-off analysis & & \\
\hline 3. To promote the development & 3.1 & Stakeholder engagement and capacity building process \\
of socially inclusive, equitable & 3.2 & Principles and pilot interventions which could underpin equitable value chains \\
and sustainable agricultural & 3.3 & Best practice guidelines for NGOs and CBOs \\
intensification policies and & 3.4 & Document key research approaches and develop accessible tools for other researchers \\
engagement processes & 3.5 & Engage policy makers and decision makers \\
\hline
\end{tabular}

Marginalised farmers in the SIAGI villages - landless, tenant, smallholder or women-headed households have struggled to achieve enough agricultural production to meet their various livelihood needs (e.g. income, nutrition, housing, education, health, and comfort). The sister projects include on-farm research to test new or improved agricultural practices, whilst the SIAGI project is focused on institutional, social, market and integration research underpinned by ethical community engagement (ECE). Prior to the commencement of the ACIAR projects, most households produced one paddy crop in the wet season and typically did not produce crops in other seasons due in part to a lack of available freshwater. The ACIAR projects have tried to help farmers address this through technical and social interventions to develop irrigation infrastructure (solar pumps and wells and surface water storages in West Bengal, and canal re-excavation in Bangladesh) and facilitate a mindset of collective actions and inclusive local water governance. 


\subsection{Inclusion and Ethical Community Engagement}

In SIAGI, inclusion is considered across gender and class (economic or social) lines. It reflects people's involvement in institutions and the implementation of activities or interventions; it also considers the material (e.g. access to resources) or intangible (e.g. appreciation, respect) benefits they receive for their contribution, and the perceived impact of these benefits (Mishra et al. 2018).

In each of the case study locations, our local NGO partners act as facilitators between the project teams and the local community. Early in the project, the proposed methodology for the SIAGI project was challenged by our NGO partners as being geared towards the needs of the researchers in that the activities were focused on eliciting data and information that we wanted for our research. We had initially anticipated several discrete tasks such as focus group discussions, household surveys, or rapid rural appraisals to collect information for the objectives and activities outlined in Table 1. Our NGO partners reasoned that this was extractive in nature, would not give back to communities and placed the project at risk of not being able to develop the trust and relationships needed to answer the research questions, let alone achieve the impact we desired for communities. In their experience working with these (and other) communities, many community members had been ignored or let down by past engagement with researchers or government stakeholders. Thus, our NGO partners argued that we should change to an ongoing engagement process where we consciously aim to balance what we provide to community partners and what the project receives in terms of data and learning. It was realised that this process would need to be coordinated within the SIAGI team as well as with the sister projects so that we could manage demands placed upon communities and avoid 'stakeholder fatigue'. It would also need to be adaptive and driven by community choices and aspirations as well as their needs. By agreeing to this change, the SIAGI team recognised community members as partners and collaborators (not research subjects), with their own perspectives, wisdom and priorities, and committed to take collective decisions about project interventions.

From this perspective, the SIAGI team set out to conceptualise ECE by distilling the behaviours and practices which enable NGO practitioners to engage more effectively. These enabling conditions for inclusive engagement, combined with core underlying values were distilled into several high-level principles. Essentially, the ECE principles outline 'the why' of ECE. In time, a list of ECE practices was generated to help orient external partners to the ECE approach and highlight practical examples of ECE in action. ECE practices have the purpose of guiding the engagement practitioner to 'the how' of ECE.

The decision to embrace an ECE approach has meant a greater reliance on qualitative methods to create dialogue with farmers, co-develop ideas, interventions and plans, and record the information needed by researchers. More quantitative methods (such as household surveys) could be used to target specific information needs but would need to be carefully designed to achieve the desired goal without placing unreasonable burden upon farmers. The implications for the integrated assessment (IA) component of the SIAGI project are outlined in Section 3.

\section{INTEGRATED ASSESSMENT}

A major component of the SIAGI project proposal was to develop and apply modelling tools that enabled the integration of data from the SIAGI and sister projects and to use these models to explore trade-offs of agricultural interventions and explore options that might offer better outcomes for marginalised households. The IA has drawn on analyses and learnings from all the SIAGI project activities: value chain analysis, bioeconomic modelling, livelihood analysis, development of a social justice framework, and policy and institutional analysis. These activities drew on a range of primary and secondary data sources and methods including narratives, focus group discussions, regional market evaluations, and desktop analysis of policy documents and environmental literature. Figure 1 gives an overview of key outputs from the IA work to date.

\subsection{Synthesising the risks and opportunities of agricultural intensification}

About six months into the project, the SIAGI team committed to ECE and chose to slow down and redesign the way in which we address the objectives and activities in Table 1. Greater emphasis was placed on identifying the immediate priorities of community members, which may not directly relate to the project aims of social inclusion in sustainable agricultural intensification. However, addressing these helped build trust between the communities and the SIAGI team and in turn this facilitated dialogue and actions related to the project (Carter, 2018; SIAGI, 2018a).

For the IA activities, two main rounds of conceptual modelling were conducted. Prior to the commitment to ECE we had developed a simple conceptual framework that enabled perspectives and processes to be mapped 
out using a common 'language' and used it to map out our partners early understanding of the agricultural and social systems in the study villages (Hamilton et al. 2017). In the second round of conceptual modelling, the project team used this framework to develop a second round of conceptual diagrams that captured their learnings from the project to that point in time.

We had initially anticipated conducting a formal, quantitative, risk assessment drawing primarily on outputs from activities 1.1 to 1.6 in Table 1. However, this approach was no longer supported once we adapted our methodologies to fit within an ECE approach and suit the use of primarily qualitative data. The outputs from the conceptual modelling, with follow-up meetings with partners in March 2018 to clarify concepts and relationships, allowed us to qualitatively synthesise the various risks and opportunities of agricultural intensification in the SIAGI case study areas (SIAGI, 2018b).

Over the last 12 months we have consolidated our research into an overarching Integrated Assessment (IA) framework that comprises three main components (see Hamilton et al. 2019, this proceedings):

- Local water management: links local water governance and community engagement to the distribution and use of these resources within the communities, and desired outcomes,

- Inclusive value chain analysis: agricultural production and marketing are linked to farm income and desired outcomes, and

- Empowering change: pathways of motivation, self-efficacy, agency and access to resources are used to represent empowerment as a process of change.

The frameworks themselves are generalised but their application is context and situation dependant. They were designed as learning tools for project teams to test ideas and assumptions around agricultural interventions through the use of narrative and/or model-based applications.

\subsection{IA model approach}

In the project proposal, we planned to select the modelling approach by month 21 (March 2018) although had considered the Bayesian Network approach to be a likely contender. Bayesian Networks were used successfully in a previous ACIAR project to study trade-offs in livelihood and resilience to drought in the context of watershed development, and we considered the approach well suited to using both qualitative and quantitative data to structure and populate the model.

The commitment to ECE encouraged us to explore how social processes and outcomes have been represented in model-based integration science for development projects. The application of Fuzzy Cognitive Mapping (FCM) approaches across the organisational behaviour (Craiger et al. 1996), livelihood analysis (Murungweni et al. 2011) and participatory modelling (Henly-Shepard et al. 2015) literature pointed to the potential of this being the most suitable approach for exploring complex social concepts with the team and our partner communities. This was supported by initial trials of the Mental Modeler software (http://www.mentalmodeler.org/, accessed 30/09/2019) with SIAGI partners, and prior positive experience with the use of a similar approach in other projects. We are now using FCM to develop semi-quantitative applications of some of our IA frameworks.

Early on we had intended to build models or frameworks specific to each case study, as the focus of the Bangladesh and Cooch Behar villages seemed quite discrete (local water management and collective farming, respectively). However, as we progressed and commonalities between case studies emerged, it became apparent that we can largely generalise the frameworks at least across the SIAGI study villages, and we hypothesise to other similar socio-agricultural settings.

\subsection{Partner participation in development process}

Given the complexity of the study systems, we intended for the whole project team to be engaged in the model development process in some form. However, the way in which partners participate in the development of the IA frameworks and associated applications (be they narrative-based or FCM) changed from our original plan. We had envisioned one to two persons each in Bangladesh and India would be trained as an integrated modeller' and would take on some responsibility for developing and using the models. Instead we have had a more even spread of direct contributions from the team into the development of the frameworks and their applications, facilitated by the Australian research partners. Our NGO partners have facilitated engagement with our community partners for the integration research. Rather than directly using the frameworks or models, our partners have discussed with the communities the issues or concepts we wanted clarification on, or used the frameworks to structure discussions or interviews. 


\subsection{Social inclusion in our frameworks and IA practices}

Capturing inclusion was critical in the both the IA frameworks and their applications using FCM or narratives. For example, in accordance with the definition of inclusion in Section 2.2, we have explicitly represented marginalized farmers participation (or not) in institutions in the local water management framework, activities to develop and maintain water resource infrastructure, and the impacts on availability of freshwater resources and marginalised farmers access to those resources. Although we developed the frameworks to be generic across SIAGI study villages and across gender, economic and social categories, the applications for each framework are context specific. The pathways from interventions to outcomes will differ between individuals or groups (esp. poor and marginalised households) as well as for different interventions. Considering gender as an example, women in the case study villages have traditionally been excluded from participating in agricultural institutions and extension activities and so their development of knowledge and skills for agriculture has been constrained. Together with limited access to physical or financial resources and their household duties, this means women farmers face many barriers to improve their agricultural production.

Informed by the principles and practice of ECE (Section 2.2), the concept of empowering change is now central to our IA work. Not only is it one component of the framework, it is intrinsically part of the inclusive value chain analysis and local water management frameworks and both of these frameworks are designed to be used conjunctively with the empowering change framework. The ECE process endeavours to enable the inclusion of individuals and groups in collaborative and participatory decision-making (SIAGI, 2018a). Marginalized groups or individuals are part of a village and broader community that can either support or hinder them in relation to the interventions of the ACIAR projects or more generally in their life activities. Capturing some of these individual and group interactions in an IA has been a key challenge that we are explicitly addressing.

\section{REFLECTIONS}

The research experience of the SIAGI project team is diverse, covering agricultural science, ethics of development, rural development and natural resource economics, poverty studies, value chain analysis, markets for the poor, bioeconomic modelling, climate risk assessment, social psychology, socio-economic impact assessment, livelihoods analysis, institutional analysis, natural resources management, integration science, qualitative and quantitative research, environmental modelling, and nutrition sensitive agriculture. Bringing in the practitioner experience - (e.g.) process facilitation, community mobilisation, strengthening community based organisations - have brought many opportunities and challenges for integrated assessment in this project.

\subsection{Outcomes of being socially inclusive in our modelling practices}

Three main outcomes have been realised from the development and application of the IA frameworks thus far. Firstly, it has been a useful learning and knowledge synthesis tool for the team. It has helped obtain conceptual convergence within the team and formalise our understanding of the complex system(s) we are dealing with. Secondly, the frameworks are discussion and learning tools that can be used to test implications of system interventions or perturbations and explore how we can improve outcomes in the community. The flipside of this is the third outcome of the IA, which is their use as a tool to check for unintended or adverse outcomes. Scenario analysis can be applied to the frameworks to ensure interventions do not lead to any adverse outcomes within the community - this is in line with the ethics principle of do no harm.

The process to developing the IA frameworks has complemented the ECE approach. In SIAGI, the ECE approach underpins both the frameworks and the process we used to develop them. By their definition, IA and ECE both look to draw on multiple perspectives, give a voice and respect to participants, and iteratively and collaboratively develop ideas and achieve better outcomes. IA can help with discussing, framing, defining, understanding socio-agricultural problems, which complements many of the processes of doing R4D ethically.

As the IA has progressed, there has been a convergence between it and the project's Theory of Change. Often, IA can be a somewhat academic exercise and its implication for practice can seem inscrutable to others - a common pitfall of complex systems research. If an IA process is reflective and focused on both outcomes (i.e. captures finite and measurable change) and impacts (i.e. longer term effects of outcomes), then it should help the team focus on what makes a difference, moving IA beyond the academic to add real value to projects.

The SIAGI project has provided us an opportunity to push representation of social processes and concepts in frameworks beyond the current state-of-practice of integrated modelling. Stojanovic et al. (2016) in a literature review critiquing social-ecological systems research, noted that "resource extraction, population, and material benefits receive greater consideration [in socio-ecological systems models] than values, equity, non-material and psychological aspects of well-being”. Our experience from SIAGI is that explicitly incorporating such 
concepts into the integrated frameworks and FCM applications was feasible and has allowed us to engage effectively with our partners working in this space. In using the frameworks, there is little risk that social processes and concepts can be sidelined when thinking through interventions, given that they are central to the pathways from intervention to outcomes.

\subsection{Learnings}

The SIAGI project has been a huge learning experience, including for the modellers whose learnings have included applying a modelling approach new to them (i.e. FCM), more in-depth use of qualitative data to inform the model structure and parameterize the model, and working with researchers in fields new to the modellers (value chain analysis, social justice, psychology, nutrition sensitive agriculture). Observing the NGO partners' community ECE practices in the field provided practical learnings that are translatable for participatory modelling (e.g. structuring discussions with communities without leading questions, observing body language, culturally appropriate engagement).

IA in SIAGI has been resource intensive but is now bearing fruit. The challenges of integration recognised in the literature hold strong, namely bridging divergences across the domains. Some of these divergences are technical (e.g. methodologies and tools) whilst others reflect different conceptual understandings arising from alternate experiences, perspectives or knowledge frames. It has taken a long time to distil key concepts and pathways to represent what are very complex social systems.

At times it proved challenging to communicate how our collective understanding has developed, although over the last 12 months this challenge has diminished as we have approached (but not yet attained) "requisite simplicity" (see Stirzaker et al. 2010). In part this reflects the time and mental effort needed to properly discuss integration and the reality of it being one of many items for discussion at the whole-of-team six monthly meetings. Early on, project meetings focused on activities that looked at specific component research, and IA discussions were necessarily constrained due to both time restrictions and our limited whole-of-system understanding. At this early stage, we were exploring the complexity of the system, including the divergent views, and therefore discussions on IA seemed somewhat confused and abstract. We had to continue to ask questions to try and gain the clarity across disciplines to develop a common and deeper understanding. It was not until we had a better understanding of what concepts and interactions we wanted to address and the learnings from the component research emerged that we were better equipped as a team to discuss the IA in greater depths. For example, during the reflection session at the conclusion of the project team meeting in early 2018, one member of the SIAGI team identified that integrated modelling itself is challenging for the research team due to their limited exposure to integrated modelling, which was made more difficult given the types of questions SIAGI is attempting to answer. Over time team members realised that they did not have to be fully versed in integrated modelling to be able to participate and provide input in the IA activities.

There is a particular challenge in trying to develop 'general' frameworks with key concepts and linkages. Typically, we do not want the detail which may be specific to location, actors, etc and this can be challenging for disciplinary experts as it may seem like we are over-simplifying their disciplinary content. Researchers in country are not used to integration and primarily specialise in domains of expertise, as is expected by their institutions. This is also evident in researchers in Australia, though there is slow shift in attitudes towards integration science. For non-IA researchers in the project, being part of conceptualising and developing frameworks provided avenues to bridge silos across disciplines, as well as push scientific boundaries in otherwise mature domains. Achieving buy-in from the whole team has been critical, but until we could build context and situation specific applications (of the generic frameworks) it was challenging to identify the key concepts.

It has been personally rewarding to have demonstrated buy-in to integration science across the project team. Most recently, our NGO partner working with the two communities near Cooch Behar, used the empowering change component framework to frame their discussions with individual community members or farmer groups to document their personal changes stemming from technical and institutional interventions aimed at creating opportunities for dry season agriculture. Over time, we have had increased engagement with IA in project workshops. As the focus of the IA became clearer (from March 2018 onwards in Figure 1) we are seeing discussions go overtime with contributions across the team (e.g. NGO, academics, students). Team members are recognising their words in the IA frameworks and being able to use and generate narratives in our work has also helped us engage with partners who have little background in systems thinking or modelling. Language will probably always be a challenge (in all integration projects not just SIAGI) but as our collective understanding of the systems developed, these issues have reduced. 
The reliance on qualitative methods and inputs has forced critical thinking and questioning of the IA framework structure, in particular ascertaining which concepts were 'general' or context specific. It has allowed us to give equal weight to social processes and concepts that are not easily quantified but which are undeniably critical in achieving goals around development, maintenance and distribution of resources, and fair access to institutions.

The SIAGI project has always had a team culture of being responsive, adaptable and integrative in nature, more so than most projects we have worked on to date. The resolution to commit to ECE and redesign all aspects of the research, was a big decision. It required a trusted and experienced project leader who had legitimacy to steer the project in the direction it needed to go. All academic and NGO partners needed to be on-board with the decision and willing to change the way in which they conducted research or engaged with communities and stakeholders. This was not without risks in terms of the expectations or accepted practices of their workplace. A flexible work plan was critical for the whole project; in the IA we had purposively taken a deliberative approach to the scoping and problem framing phases. This caution proved to be well founded as we had not expected to choose the FCM or narrative-based approaches as the tools for applying the integrated assessment frameworks we have developed. Having the time and considerable support from the project team enabled us to adapt the IA approach to fit with ECE and effectively synthesise SIAGIs learnings.

\section{ACKNOWLEDGMENTS}

The SIAGI (Promoting socially inclusive and sustainable agriculture intensification in West Bengal and Bangladesh) project (LWR/2014/072) is funded by the Australian Centre for International Agricultural Research (ACIAR) and is led by the Commonwealth Scientific and Industrial Research Organisation (CSIRO). We acknowledge contributions from all members of the SIAGI project team to the integration activities and thank them for their continued engagement, insights to and trust in what has at times been a challenging and uncertain path to integration. The CSIRO Ethics Clearance reference for the SIAGI project is 083/15.

\section{REFERENCES}

Carter, L. (2018) Ethical engagement for research partnerships and collaboration. Development Bulletin, 79, 49-52.

Craiger, J.P., Goodman, D.F., Weiss, R.J. Butler, A. (1996). Modeling organizational behavior with fuzzy cognitive maps. International Journal of Computational Intelligence and Organizations, 1, 120-123.

Hamilton, S.H., Merritt, W.S. Carter, L, Lim-Camacho, L. Nidumolu, U., Cosijn, M., Mishra, R., Dash, M., Roth, C. (2017). Developing a common language for transdisciplinary modelling teams using a generic conceptual framework. MODSIM 2017, 22nd International Congress on Modelling and Simulation. Modelling and Simulation Society of Australia and New Zealand, December 2017, pp. 1447-1453.

Hamilton, S.H., Merritt, W.S., Lim-Camacho, L., Carter, L., Cosijn, M., Roth, C.H. (2019) Integrated assessment frameworks for understanding pathways for socially inclusive agricultural intensification. MODSIM 2019, 23rd International Congress on Modelling and Simulation. Modelling and Simulation Society of Australia and New Zealand, December 2019.

Henly-Shepard, S., Gray, S.A. and Cox, L.J. (2015). The use of participatory modeling to promote social learning and facilitate community disaster planning. Environmental Science \& Policy, 45, 109-122.

Mishra, R., Ray, D., Mishra, P, Ghosh, M, Majumdar, S. (2018). Ethical community engagement for intensification, inclusion and collectivization. SIAGI Project Report, July 2018, https://siagi.files.wordpress.com/2018/07/siagi-report ethical-community-engagementcollectives final july-2018.pdf

Murungweni, C., Van Wijk, M.T., Andersson, J.A., Smaling, E.M.A., Giller. K.E. (2011). Application of fuzzy cognitive mapping in livelihood vulnerability analysis. Ecology and Society, 16: 8.

SIAGI (2018a). Process, principles and challenges of inclusive community engagement: distilling NGO best practice. SIAGI Project Report, July 2018, https://siagi.files.wordpress.com/2018/07/siagi-report ngopractice -report-for-mtr final july-2018.pdf.

SIAGI (2018b). A synthesis of risks and opportunities of agricultural intensification in the SIAGI case study areas. SIAGI Project Report, July 2018, https://siagi.files.wordpress.com/2018/07/siagireport_dsi_assessment_final_july-2018.pdf.

Stirzaker, R., Biggs, H., Roux, D. Cilliers, P. (2010). Requisite simplicities to help negotiate complex problems. Ambio, 39, 600-607.

Stojanovic, T., McNae, H., Tett, P., Reis, J., Smith, H.D. Dillingham, I., (2016). The "social” aspect of socialecological systems: a critique of analytical frameworks and findings from a multisite study of coastal sustainability. Ecology and Society 21, 15. 